Copyright (C) 2014 IEEE. Personal use of this material is permitted. Permission from IEEE must be obtained for all other uses, in any current or future media, including reprinting/republishing this material for advertising or promotional purposes, creating new collective works, for resale or redistribution to servers or lists, or reuse of any copyrighted component of this work in other works. 


\section{On the Location of Plug-In Relay Devices for Indoor Power Line Communication Environment}

\author{
Xiaolin $\mathrm{Wu}$ \\ Dept. of Electrical and Computer Engineering \\ Curtin University of Technology \\ Perth, Australia \\ xiaolin.wu@curtin.edu.au
}

\author{
Yue Rong \\ Dept. of Electrical and Computer Engineering \\ Curtin University of Technology \\ Perth, Australia \\ Y.Rong@curtin.edu.au
}

\begin{abstract}
Indoor power line communication (PLC) systems experience high attenuation and frequency-selective channel condition, in addition to different types of colored noises. Its performance is heavily influenced by the length and topology of the in-house power cable wiring. By exploiting the broadcast property of the indoor power grid to each outlet, this paper presents a simple but practical relay-based cooperative communication scheme for indoor PLC networks. By plugging a relay device into a third outlet between the transmitter and receiver, reliable signal-to-noise ratio (SNR) can be cooperatively achieved by setting proper system parameters. The condition for the optimal location of the plug-in relay device has been identified. Numerical simulation results show that with the cost of extra relay device, more reliable performance can be obtained with respect to the conventional direct transmission scheme.
\end{abstract}

Keywords- Power Line Communication (PLC); Cooperative Communication Scheme; Plug-in Relay Device

\section{INTRODUCTION}

With the worldwide existing power grid infrastructure, power line communication (PLC) technology has received a renewed attention in the last decades. At the same time, applications based on this technology attract a lot of commercial interests, such as Smart Grid, automatic meter reading (AMR), advanced metering infrastructure (AMI), etc. For the indoor environment, in addition to electricity delivery, power grids are also often used as medium to support local area networks (LAN). However, this type of indoor PLC channel has demonstrated a hostile characteristics for broadband communications [1], which mainly consist of four aspects: (1) high attenuation in high-frequency (HF) band; (2) frequencyselective fading in all available band; (3) time-varying property with different time scales; (4) considerable non-white Gaussian noises.

A lot of efforts have been made in order to apply advanced technologies, which were originally developed for wireless environment, into PLC channels. Some of them, like Windowed OFDM and Turbo Coding, have been included in the current IEEE1905 standard for indoor PLC networking [2]. Research on introducing diversity into PLC has also being undertaken. Considering that indoor power grid cables usually consist of three conductors, namely Line, Neutral, and Protective Ground cables, every two of them can be seen as an independent port i.e. sub-channel, so that a low diversity order multiple-input-multiple-output (MIMO) system can be realized if all three conductors are used at the same time [3].

On the other hand, with a single pair of transmission wires, the idea of cooperative communication can be adapted into the indoor PLC environment, usually by plugging relay devices into the outlets located between the transceivers. Due to the broadcasting nature of the power cables, these relay devices may receive and forward the source message to the destination. In [4], the optimal time-slot duration allocation between the direct transmission phase and the relay phase has been investigated. Under the assumption that each network node is a potential relay node, [5] proposed a multi-hop transmission scheme combined with the application of distributed spacetime block coded (DSTBC) in PLC networks.

Different to the approach in [5], by considering both the direct and multi-hop relay transmission effect, in this work we propose a simple but practical relay-assisted transmission strategy for indoor PLC environment. Our main contributions are as follows. Firstly the performance improvement of the cooperative amplify-and-forward (CAF) scheme has been investigated with respect to the conventional direct transmission (DT) system. In addition, to the best knowledge of the authors, this is the first time that the general condition for the optimal location of the plug-in relay device has been identified.

The rest of this paper is organized as follows. Section II describes the indoor PLC wiring topology and its corresponding channel and noise model. Following this, the CAF system has been analyzed in Section III. Based on maximizing the effective signal-to-noise ratio, the condition of the optimal location of the relay node has been given. Section IV presents simulation results under a typical configuration of indoor PLC channel environment. The performance of CAF scheme has been examined and compared with respect to the DT system. Finally, Section V concludes the paper.

\section{SYSTEM MODEL}

\section{A. Indoor PLC network topology}

Fig. 1 shows a typical arrangement of the power grid in a house, which allows us to understand how relay scheme can be practically applied. Under this arrangement, every pair of outlets/sockets can be employed as a point-to-point 
communication system, and its corresponding channel is characterized by the wiring topology and load impedances between the transceivers.

For example, here we choose message source $(S)$ and destination $(D)$ outlets respectively. We refer to the shortest link between the transceivers as main path and its length as main length; other wirings are as treated as tap-brunches attached to the main path, which contribute to the multi-path distortion effect of the channel. Any outlet located on this main path (e.g. $R_{1}$ ) or on a brunch of this main path (e.g. $\left.R_{2}\right)$ can be deployed with a plug-in relay device, which we will refer to as the relay node. Let us also denote the channel between $x$ and $y$ as $H_{x y}$ and its transfer function as $H_{x y}(f)$, where $\{x, y\} \in\left\{\{S, D\},\left\{S, R_{1,2}\right\},\left\{R_{1,2} D\right\}\right\}$. From a relay node's point of view, the whole direct channel has been separated into two parts, i.e. the source-to-relay channel and the relay-todestination channel. Then it is obvious that

$$
H_{S D}(f)=H_{S R_{1}}(f) H_{R_{1} D}(f) .
$$

This relation has been illustrated in Fig. 2 .

Now, we assume a signal $X(f)$, with its power spectral density (PSD) as $P S D_{X}(f)$, is transmitted through $H_{S D}$. The output signal $Y(f)$ has a PSD as $P S D_{Y}(f)$. Then we have

$$
P S D_{Y}(f)=P S D_{X}(f)\left|H_{S D}(f)\right|^{2} .
$$

If we denote the power of $X(f)$ and $Y(f)$ respectively as $P_{X}$ and $P_{Y}$, and the channel's bandwidth is from $f_{\min }$ to $f_{\max }$, then

$$
\begin{aligned}
P_{Y} & =\int_{f \text { min }}^{f \max } P S D_{Y}(f) d f \\
& =\int_{f_{\min }}^{f_{\max }} P S D_{X}(f)\left|H_{S D}(f)\right|^{2} d f \\
& =\left|H_{S D}(\Omega)\right|^{2} \int_{f_{\min }}^{f_{\max }} P S D_{X}(f) d f, \quad f_{\min }<\Omega<f_{\max } \\
& =\left|H_{S D}(\Omega)\right|^{2} P_{X}, \quad f_{\min }<\Omega<f_{\max } .
\end{aligned}
$$

Note we used the first mean value theorem for integration in the derivation of (4). Actually, $\left|H_{S D}(\Omega)\right|^{2}$ is a metric for the average power attenuation of $H_{S D}$. Similarly we can write

$$
\begin{aligned}
& P_{Y}=\int_{f_{\min }}^{f_{\max }} P S D_{X}(f)\left|H_{S D}(f)\right|^{2} d f \\
&=\int_{f_{\min }}^{f_{\max }} P S D_{X}(f)\left|H_{S R_{1}}(f)\right|^{2}\left|H_{R_{1} D}(f)\right|^{2} d f \\
&=\left|H_{S R_{1}}(\Phi)\right|^{2} \int_{f_{\min }}^{f_{\max }} P S D_{X}(f)\left|H_{R_{1} D}(x)\right|^{2} d f, \quad f_{\min }<\Phi<f_{\max } \\
&=\left|H_{S R_{1}}(\Phi)\right|^{2}\left|H_{R_{1} D}(\Theta)\right|^{2} P_{X}, \quad f_{\min }<\Phi<f_{\max } \\
& f_{\min }<\Theta<f_{\max }
\end{aligned}
$$

Let us denote $\left|H_{S D}(\Omega)\right|^{2},\left|H_{S R_{1}}(\Phi)\right|^{2},\left|H_{R_{1} D}(\Theta)\right|^{2}$ as $\alpha_{S D}, \alpha_{S R_{1}}$, $\alpha_{R_{1} D}$ respectively. Then from (4) and (5), it is obvious that

$$
\alpha_{S D}=\alpha_{S R_{1}} \alpha_{R_{1} D}
$$

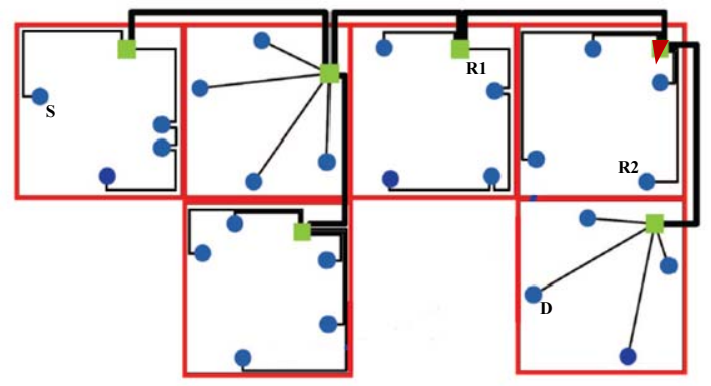

Fig. 1. A typical indoor power cable wring topology showing main panel (triangle), derivation boxes (square) and connections with outlets/sockets (circle) [5].

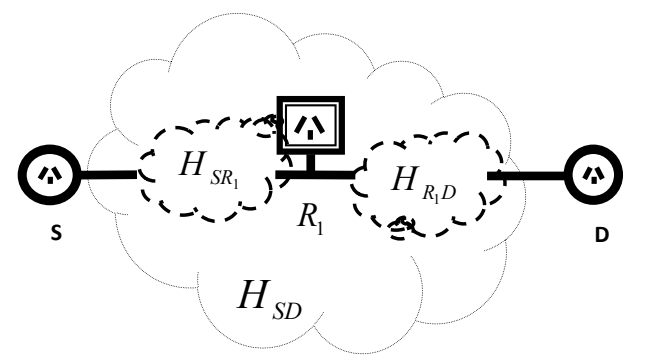

Fig. 2. A relay-assisted indoor PLC system.

\section{B. Channel modelling}

It is not only the power grid deployment practice varies in different parts of the world, but also in a particular network, the selection of the outlets to deploy the transceivers has a strong influence on the final channel response characteristics [1]. These factors make a generally accepted channel model still not available. As an alternative, some statistical models have been proposed based on extensive measurement campaigns. An excellent one among them was suggested in [6].

Applying the channel generator in [7], randomly generated channels can be used as source-to-destination, source-to-relay and relay-to-destination channels respectively in our system. Examples of these channels are shown in Fig. 3 by their frequency responses.

As observed in Fig. 3, deep frequency-selective distortion usually appears in PLC channel. This may cause low reliability in some frequency bands due to their very high attenuation and sharply changing slopes. In addition, the authors of [8] reported that PLC channel can be viewed as a linear periodical time varying (LPTV) system. However, considering its relative long coherent time, i.e. about $600 \mu$ s, we can reasonably treat it as quasi-stationary channel in this work.

\section{Noise modelling}

The noise in PLC has been studied in [9] and [10]. In general they can be classified into two classes: general coloured background noise (GBN) and impulsive noise. The GBN is the sum of several low power noise sources and narrowband interference which are introduced by medium and short wave broadcasts. The GBN often has a stable PSD over seconds, minutes and even hours. In [10], it is reported that this PSD is not white, but in any narrow band the noise's probability distribution function (PDF) can be readily approximated as Gaussian. On the other hand, the impulse 
noise may change rapidly within microseconds and milliseconds.

In this paper, we only consider the impact of the GBN. Based on extensive measurements, a synthesis process which passes the white Gaussian noise (WGN) through a coloured filter is used to approximate the general background noise [11]. An example of this noise is given by its PSD in Fig. 4.

\section{OPTIMAL RELAY LOCATION}

We assume that the time division duplexing (TDD) mode is used in our scheme, and coherent signal combination is used at the receiver. We also assume that the channel state information (CSI) is known at both the relay and destination nodes. As the PLC system can cause interference to other radio receivers in the shortwave range, in order not to cause more electromagnetic interference (EMI) to the environment, the total power consumption of relay and transmitter in our schemes is assumed to be equal to the transmission power of the DT system.

Based on above system configurations, let us consider the scenario where a relay node (e.g. $R_{1}$ ), working in amplify-andforward mode, assists the data transmission from the source to destination nodes. This has been illustrated in Fig. 5.

At the first time-slot, the source sends message signal $X(f)$ out with power $P_{s}$. The relay and the destination receive this signal with different channel attenuations and noises. In the frequency domain, they can be written as

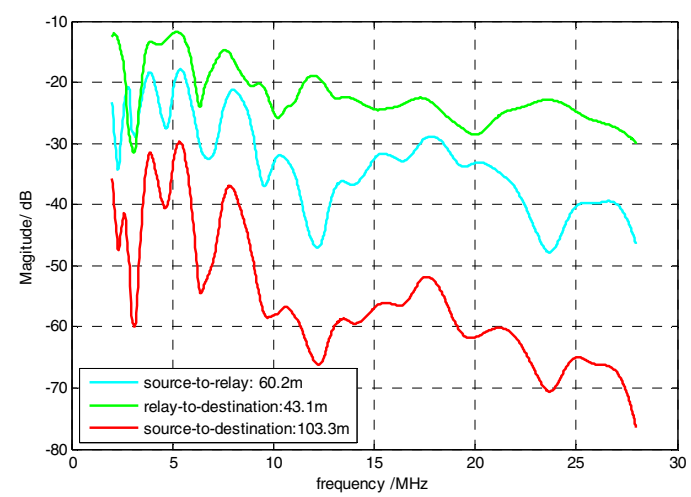

Fig. 3. Magtitude spectra of three example randomly generated PLC channels, with their main lengths indecatied.

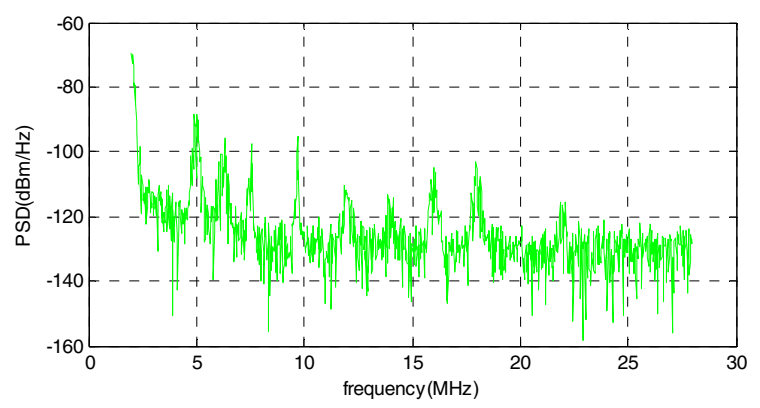

Fig. 4. PSD of GBN in PLC channel.

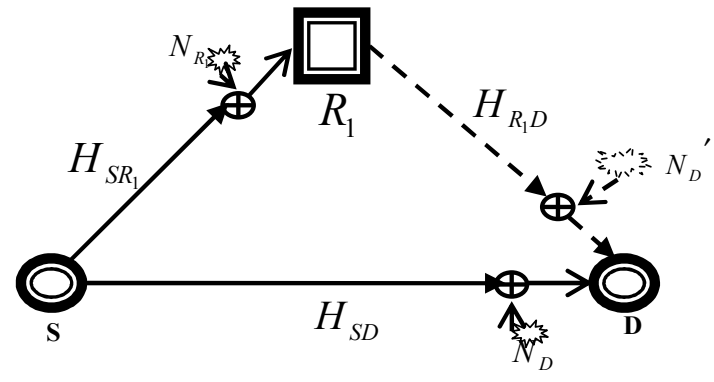

Fig. 5. Source node (S) transmits information to the destination node (D) with the aid of a relay node (R1). Dash line emphasizes the TDD mode.

$$
\begin{aligned}
& Y_{R_{1}}(f)=H_{S R_{1}}(f) X(f)+N_{R_{1}}(f) \\
& Y_{D}(f)=H_{S D}(f) X(f)+N_{D}(f) .
\end{aligned}
$$

The signal-to-noise ratio (SNR) at the destination is

$$
S N R_{D}^{<1>}=\frac{P_{s} \alpha_{S D}}{P_{N_{D}}}
$$

where $P_{N_{D}}$ is the noise power at destination.

At the second time-slot, the relay node amplifies its received signal $Y_{R_{1}}(f)$ with power gain $\beta$, and forward it to the destination with power $P_{r}$. Thus the received signal at the destination at this phase is written as

$$
\begin{aligned}
Y_{D}^{\prime}(f) & =\sqrt{\beta} Y_{R_{1}}(f) H_{R_{1} D}+N_{D}^{\prime}(f) \\
& =\sqrt{\beta} H_{S D}(f) X(f)+\sqrt{\beta} H_{R_{1} D}(f) N_{R_{1}}(f)+N_{D}^{\prime}(f) .
\end{aligned}
$$

As the power of signal $Y_{R_{1}}(f)$ can be calculated as $P_{s} \alpha_{S R_{1}}+P_{N_{R_{1}}}$, we have

$$
\beta=\frac{P_{r}}{P_{s} \alpha_{S R_{1}}+P_{N_{R_{1}}}}
$$

where $P_{N_{R_{1}}}$ is the noise power at the relay node. From (7), we can calculate the SNR at the destination node at this second phase is

$$
S N R_{D}^{<2>}=\frac{\beta \alpha_{S D} P_{s}}{P_{N_{D}}{ }^{\prime}+P_{N_{R_{1}}} \beta \alpha_{R_{1} D}} .
$$

Finally, the receiver combines the signals in (5) and (7) with the maximum ratio combing (MRC) technique, so that the effectively cooperative-signal-to-noise ratio $(C S N R)$ can be expressed as

$$
C S N R=S N R_{D}^{<1>}+S N R_{D}^{<2>} .
$$

Generally, the noise PSD depends on the outlet location of the power grid, e.g., an outlet near a noise-generating appliance has a higher PSD than the one which is located further. For simplicity we assume that the noise on every point of the PLC channel has the same power, i.e. $P_{N_{D}}=P_{N_{D}}{ }^{\prime}=P_{N_{R_{1}}}=P_{n}$. By using (6) and (8)-(10) we have

$$
C S N R=\frac{P_{s} \alpha_{S D}}{P_{n}}+\frac{P_{s} P_{r} \alpha_{S D}}{P_{s} P_{n} \alpha_{S R_{1}}+P_{n}^{2}+P_{n} P_{r} \alpha_{R_{1} D}} .
$$


By observing (11), we can see that the higher relay power $P_{r}$ used, the better performance improvement will be achieved. However, this trend will not last all the time, but converge to its limitation. This has been shown in (12) and Fig. 6.

$$
\begin{aligned}
& \lim _{P_{r} \rightarrow \infty} C S N R \\
= & \lim _{P_{r} \rightarrow \infty}\left\{\frac{P_{s} \alpha_{S D}}{P_{n}}+\frac{P_{s} P_{r} \alpha_{S D}}{P_{s} P_{n} \alpha_{S R_{1}}+P_{n}^{2}+P_{n} P_{r} \alpha_{R_{1} D}}\right\} \\
= & \frac{P_{s} \alpha_{S D}}{P_{n}}+\frac{P_{s} \alpha_{S D}}{P_{n} \alpha_{R_{1} D}} .
\end{aligned}
$$

In practice, we often notice that there is more than one outlet available between the transceivers. To benefit as much as possible from the relay power, we need to decide which outlet we should choose to deploy the relay device. For given $P_{n}, P_{s}$ and $P_{r}$, this problem boils down to solve the following optimisation problem.

$$
\begin{array}{ll}
\max _{\alpha_{S R_{1}}, \alpha_{R_{1} D}} & C S N R \\
=\max _{\alpha_{S R_{1}}, \alpha_{R_{1} D}} & \frac{P_{s} \alpha_{S D}}{P_{n}}+\frac{P_{s} P_{r} \alpha_{S D}}{P_{s} P_{n} \alpha_{S R_{1}}+P_{n}^{2}+P_{n} P_{r} \alpha_{R_{1} D}} \\
& 0<\alpha_{S R_{1}}<\alpha_{S D}, \quad \alpha_{S D}=\alpha_{S R_{1}} \alpha_{R_{1} D} . \\
\text { s.t. } \quad 0<\alpha_{R_{1} D}<\alpha_{S D},
\end{array}
$$

Note in (13) the first term is constant. By using CauchySchwarz inequality, it is easy to show that

$$
\frac{P_{s} P_{r} \alpha_{S D}}{P_{s} P_{n} \alpha_{S R_{1}}+P_{n}^{2}+P_{n} P_{r} \alpha_{R_{1} D}} \leq \frac{P_{s} P_{r} \alpha_{S D}}{P_{n}^{2}+2 P_{n} \sqrt{P_{r} P_{s} \alpha_{S D}}}
$$

where the equality is achieved at $P_{s} \alpha_{S R_{1}}=P_{r} \alpha_{R_{1} D}$, or equivalently

$$
\frac{\alpha_{R_{1} D}}{\alpha_{S R_{1}}}=\frac{P_{s}}{P_{r}}
$$

Thus we find (14) is the condition for the optimal location of the relay node. It indicates that theoretically to get the most benefit from the relay node, one should deploy the relay device at the outlet where the ratio of attenuations of the relay-todestination channel and source-to-relay channel is equal to the ratio of the source and relay power. However, in a realistic indoor power line grid it is not always possible to find an outlet which satisfies the above condition. But, this gives us suggestion about the best-effort choice. We will examine more details about this in Section IV.

Fig. 7 shows the comparison of CSNR (11) and $S N R_{d}^{<1>}$ (6) versus the difference between $\alpha_{R_{1} D}$ and $\alpha_{S R_{1}}$ in $\mathrm{dB}$. Note that in the DT case, SNR does not change as there is no relay node introduced. In addition to illustrating the fact of (14), it also shows us that for a given $P_{r}$, as the $P_{s}$ increasing the SNR improvement from CAF becomes less significant.

Let us consider a special case. When a relay-assisted PLC system is used on the outdoor long distance middle voltage (MV) power lines, it is custom to use the relay device to recover the power of attenuated communication signal, i.e. set $P_{r}=P_{s}$. As there is no tap-branches existing on MV power cable, the best location choice for relay device to deploy is at the middle point of the MV cable (so that makes $\alpha_{S R_{1}}=\alpha_{R_{1} D}$ ). This case has been studied in [12]. Obviously (14) generalizes the result to fading PLC channel scenario.

\section{Simulation AND NumericAl RESUlts}

In this section, we investigate the performance of the CAF scheme with respect to the DT system. To make our simulation close to the realistic situation, we refer to the HomePlug AV specification [13] and set system parameters as in Table I.

\section{TABLE I. SYSTEM SIMULATION PARAMETERS SETTTING}

\begin{tabular}{lll}
\hline Parameter & Value \\
\hline Frequency band (FB) & from 2MHz to 28MHz \\
\hline \multirow{2}{*}{ OFDM } & total subcarriers number & 1156 \\
& used subcarriers number & 917 \\
& frequency of used subcarriers & linear distributed in FB \\
\hline Modulation mode & fixed BPSK / QPSK \\
\hline \multirow{2}{*}{ Throughput } & DT system & $20.6246 \mathrm{Mbps} / 41.2492 \mathrm{Mbps}$ \\
& CAF or CDF scheme & $10.3123 \mathrm{Mbps} / 20.6246 \mathrm{Mbps}$ \\
\hline \multirow{2}{*}{ Source-to-relay channel } & main length & 17.6 meters \\
& attenuation & $-19.2126 \mathrm{~dB}$ \\
\hline \multirow{2}{*}{ Relay-to-destination channel } & main length & 46.5 meters \\
& attenuation & $-21.9342 \mathrm{~dB}$ \\
\hline \multirow{2}{*}{ Source-to-destination channel } & main length & $64.2 \mathrm{~meters}$ \\
& attenuation & $-39.0858 \mathrm{~dB}$ \\
\hline The power of GBN on channel & & $0 \mathrm{dBm}$ \\
\hline
\end{tabular}

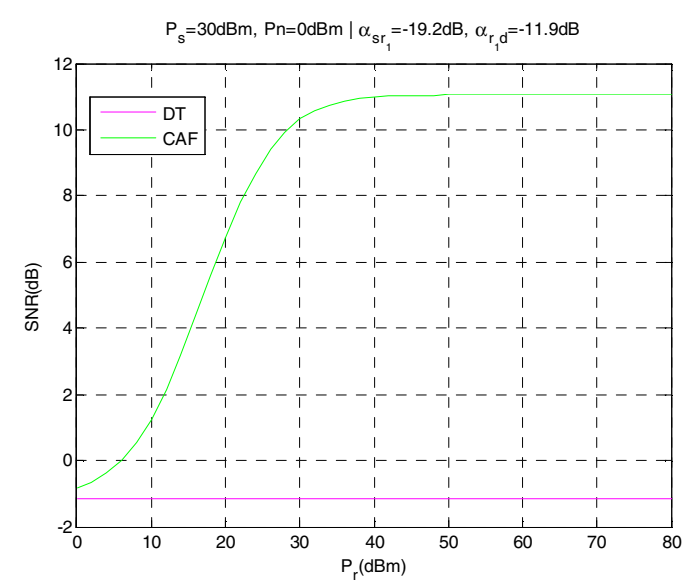

Fig. 6. Convergence of benefit from CAF with one relay node, compared with the DT scheme.

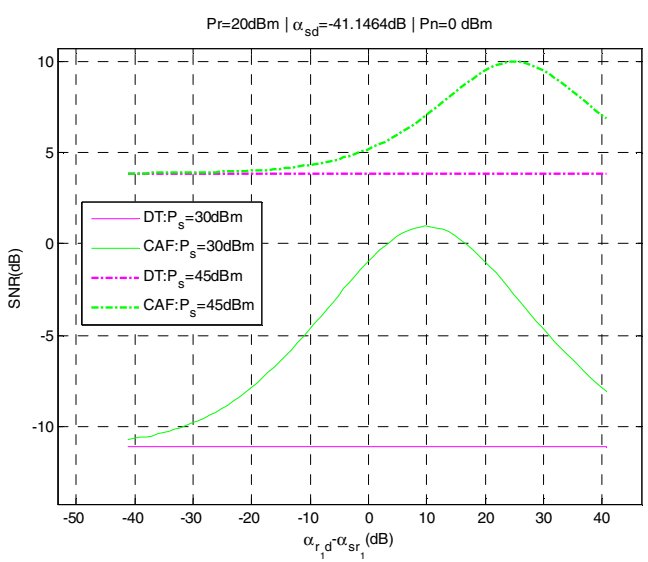

Fig. 7. SNR of the CAF scheme with varying ratio of attenuations, which is decided by relay's location, compared with the DT scheme. 


\section{A. Direct Transmission}

To give us a benchmark for comparison of our scheme, we would like to observe the bit-error-rate (BER) performance of the conventional DT system. With a randomly generated source-to-destination PLC channel, we compare the performance of the DT system with the ideal additive white Gaussian noise (AWGN) channel. The result is shown in Fig. 8.

It can be seen that due to the attenuation of the PLC channel, the BER is much higher than the AWGN channel. In addition, the different curve slopes indicate that the noise on PLC channel is not white.

\section{B. CAF Relay Scheme}

By setting $P_{r}$ to different values, we observe a group of BER versus $P_{s}$ curves as shown in Fig. 9. It indicates that the CAF scheme has a better BER than the DT system. As $P_{s}$ is increased to a high level, which means a good SNR is available on the direct path, the relay's contribution become less obvious. This observation corresponds to the conclusion drawn from Fig. 7 in Section III.

As shown in Fig. 10, by setting $P_{s}$ to different values, a group of BER versus $P_{r}$ curves can be observed. We can see that for a given $P_{s}$, the higher relay power $P_{r}$ used, the better performance improvement will be achieved until this trend converges to its limitation. This observation is corresponding to the conclusion drawn in (12).

\section{A practical example of relay location choice}

To illustrate the optimal relay node location choosing method, let us consider a practical example as shown in Fig. 11. Between the source and destination nodes, there are two available outlets $\mathrm{A}$ and $\mathrm{B}$. As we know the channels' power attention between S, A, B and D, we can use (14) as a criterion to decide which outlet we should choose to deploy the relay device for a better performance improvement.

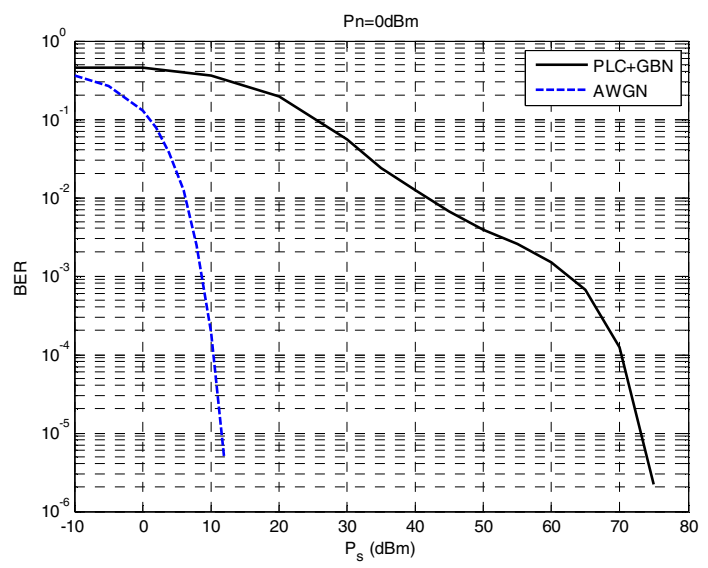

Fig. 8. Performance of DT, compared with AWGN channel with the same noise power.

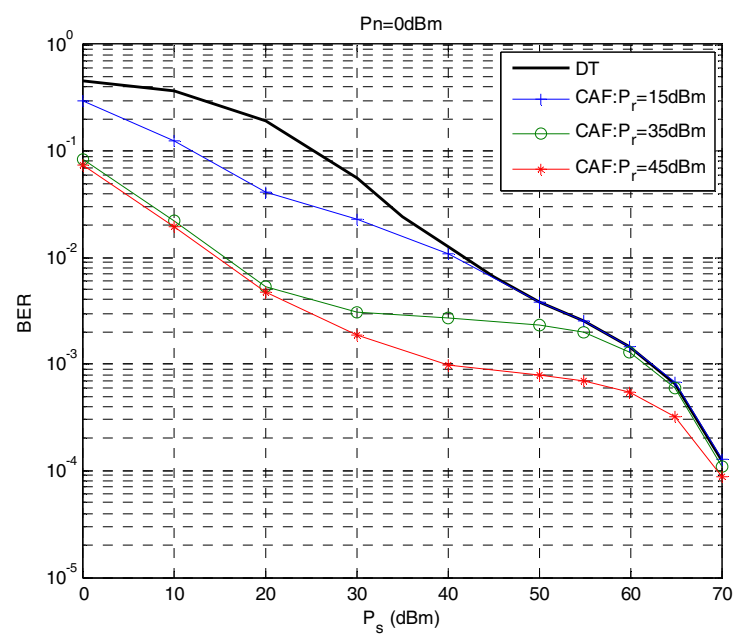

Fig. 9. Performances of CAF when different forward power used by relay node.

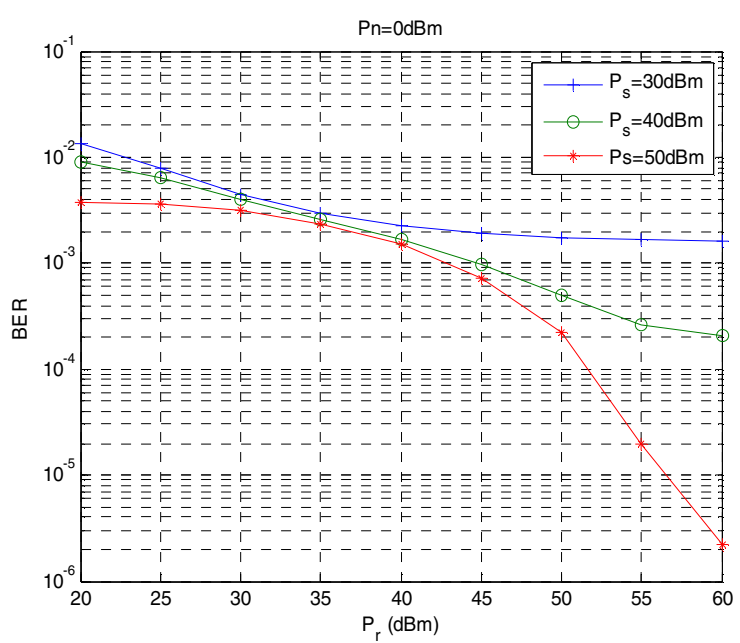

Fig. 10. Performances of CAF when source node use different transmission power.

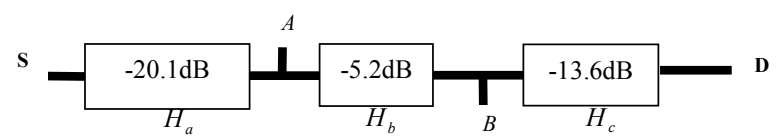

Fig. 11. A practical example of relay location choice.

Here, by assuming the source power and relay power are equal (i.e. $P_{s}=P_{r}$ ), we calculate as follows. If $\mathrm{A}$ is chosen, then

$$
\frac{\alpha_{A D}}{\alpha_{S A}}=(-5.2 d B-13.6 d B)-(-20.1 d B)=1.3 d B
$$

If $B$ is chosen, then

$$
\frac{\alpha_{B D}}{\alpha_{S B}}=(-13.6 d B)-(-20.1 d B-5.2 d B)=11.7 d B .
$$

By comparing (15) and (16), we find that the value of (15) is more close to $0 \mathrm{~dB}$. This means choosing outlet A can make the situation more close to the best condition. In other words, outlet $\mathrm{A}$ is the best-effort choice here. This has been verified by simulion result as shown in Fig. 12. 


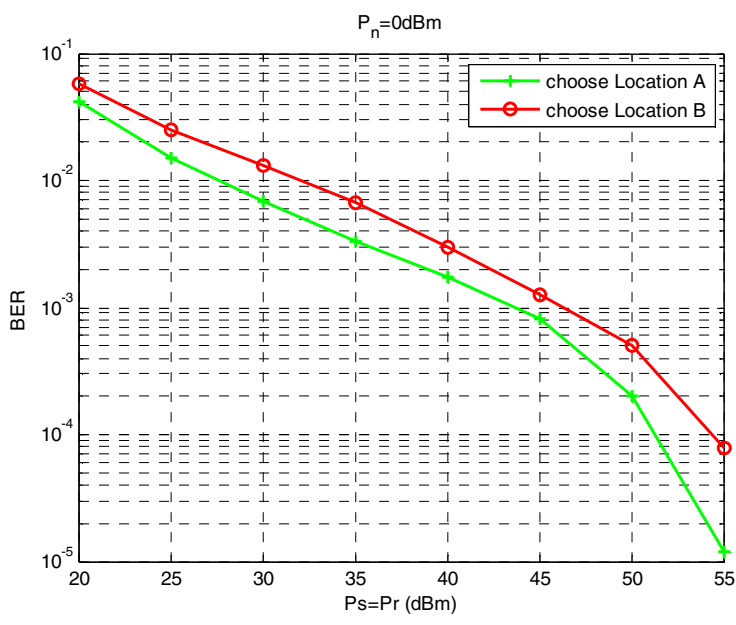

Fig. 12. Simulation result for the practical example in Fig. 11.

\section{CONCLUSION}

In this paper, we introduced a simple but practical relaybased cooperative scheme for indoor PLC networks. We present the performance improvement with respect to the conventional direct transmission system. In addition, the condition for the optimal location of the relay node has been derived. As our example shows, this condition can be used as a criterion to guide the practical technician to choose the besteffort location to deploy the relay device for an indoor PLC system.

\section{REFERENCES}

[1] H. C. Ferreira, L. Lampe, J. Newbury, and T. Swart, Power Line Communications: Theory and Applications for Narrowband and Broadband Communications over Power Lines, Wiley, 2011.
[2] H. A. Latchman, S. Katar, L. W. Yonge III, and S. Gavette, Homeplug $A V$ and IEEE 1901: A Handbook for PLC Designers and Users, Wiley, 2013.

[3] L. T. Berger, A. Schwager, P. Pagani, and D. M. Schneider, MIMO Power Line Communications: Narrow and Broadband Standards, EMC, and Advanced Processing, Taylor \& Francis, 2014.

[4] A. M. Tonello, F. Versolatto, and S. D'Alessandro, "Opportunistic relaying in in-home PLC networks", in IEEE Global Telecommunications Conference (GLOBECOM ), Miami, FL, USA, Dec. 2010.

[5] L. Lampe, R. Schober, and S. Yiu, "Distributed space-time coding for multihop transmission in power line communication networks", IEEE $J$. Sel. Areas Comm.,vol.24, no.7,pp. 1389-1400, Jul., 2006.

[6] F. J. Canete, J. A. Cortés, and L. Díez, "A channel model proposal for indoor power line communications", IEEE Comm. Mag. , vol.49, no.12, pp.166-174, Dec. 2011.

[7] http://www.plc.uma.es, website of the PLC group, ingeniería de Comunicasiones Department, Universidad de Málaga.

[8] F.J. Canete, J. A. C. Arrabal, L. Diez del Rio, and J. T. E. Munoz, "Analysis of the cyclic short-term variation of indoor power line channels", IEEE J. Sel. Areas Comm. vol.24, no. 7. pp. 1327-1338,2006.

[9] M. Zimmermann and K. Dostert, "Analysis and modeling of impulsive noise in broad-band powerline communications", IEEE Transactions on Electromagnetic Compatibility, vol.44, no.1, pp.249-258, Feb. 2002.

[10] J. A. Cortés, L. Díez , F. J. Cañete, and J. J. Sánchez-Martínez, "Analysis of the indoor broadband power-Line noise scenario", IEEE Transactions on Electromagnetic Compatibility, vol.52, no.4, pp.849858, Nov. 2010.

[11] D. Benyoucef, "A new statistical model of the noise power density spectrum for powerline communication", In Proceedings of the 7th International Symposium on Power-Line Communications and its Applications, Kyoto, Japan, pp. 136-141. 2003.

[12] X. Cheng, C. Rui, and L. Yang, "On the system capacity of relay-aided powerline communications", in International Symposium on PLC and Its Appl. (ISPLC), Udine, Italy, pp. 170-175, 2011.

[13] HomePlug Powerline Alliance, "HomePlug AV white paper." http://www. homeplug. org/products/whitepapers/ (2007). 